\title{
Bajo peso al nacer e inseguridad alimentaria en el hogar en Pasto, Colombia
}

\author{
Low birth weight and food insecurity at home in Pasto, Colombia
}

Héctor Hernán Morillo-Rosero'1 orcid.org/0000-0002-0075-9290

Maylen Liseth Rojas-Botero2* orcid.org/0000-0002-5358-6426

1 Grupo de investigación en Epidemiología y Bioestadística. Universidad CES. Medellín, Colombia

2 Grupo de Investigación Demografía y Salud. Universidad de Antioquia. Medellín, Colombia

\section{Resumen}

Introducción: El peso al nacer es un importante indicador de salud del recién nacido, de la madre y de las condiciones de salubridad de una sociedad; además es reconocido como la principal causa de morbimortalidad en la infancia. Objetivo: Determinar la asociación entre seguridad alimentaria en el hogar durante el último trimestre del embarazo y el bajo peso al nacer. Materiales y métodos: Estudio de casos y controles; se incluyeron 73 recién nacidos a término con bajo peso y 73 neonatos con peso normal. Se analizaron variables demográficas, clínicas y de seguridad alimentaria. Resultados: El bajo peso al nacer se asoció con la inseguridad alimentaria en el hogar (OR: 19,3; IC 95\%: 6,5-56,9), ajustando por hipertensión arterial de la madre, vaginosis, antecedente de ruptura prematura de membranas y malnutrición gestacional por exceso. Conclusiones: Se encontró una asociación importante entre la inseguridad alimentaria en el hogar durante el último trimestre de gestación y el bajo peso al nacer de los neonatos atendidos en el hospital San Pedro de Pasto. La fuerza de la asociación podría subestimarse en ausencia de ajuste por variables clínicas y demográficas.

Palabras clave: Recién nacido de bajo peso; seguridad alimentaria y nutricional; Colombia. (Fuente: DeCS, Bireme).

\begin{abstract}
Introduction: Birth weight is an important indicator of the health of the newborn and of the mother. It can also be used as a parameter to define sanitary conditions of societies. Low birth weight is well known as the main cause of morbidity and mortality in childhood. Objective: To determine the association between home food security during the last trimester of pregnancy and low birth weight. Materials and methods: A cases and controls study that included 73 full-term newborns with low weight and 73 normal weight neonates. Demographic, clinical and food security variables were analyzed. Results: Low birth weight was associated with home food insecurity (OR: 19.3; CI 95\%: 6.5-56.9), when adjusted to mother's arterial hypertension, vaginosis, antecedent of premature rupture of membranes and gestational malnutrition. Conclusions: We found a significant association between home food insecurity during the last gestational trimester and low birth weight of neonates born in San Pedro Hospital, Pasto. The strength of this association could be underestimated in the absence of adjustments to demographic and clinical variables.
\end{abstract}

Key words: Infant, low birth weight; food and nutrition security; Colombia. (Source: DeCS, Bireme).

\footnotetext{
*Autor de correspondencia

Maylen Rojas Botero

e-mail: maylen.rojas@udea.edu.co
} 


\section{Introducción}

El peso al nacer es un indicador pronóstico para la salud y el desarrollo del ser humano, que además señala las condiciones de salud del binomio madre e hijo(1). Se ha documentado que las condiciones al nacer repercuten en el desarrollo físico e intelectual a lo largo de la vida, de manera que un niño que nace con bajo peso tendrá mayor probabilidad de presentar dificultades en el desarrollo pleno de sus facultades ${ }^{(2-4)}$, y un mayor riesgo de muerte ${ }^{(5)}$.

El bajo peso al nacer (BPN), definido como el nacimiento de un niño con un peso inferior a $2.500 \mathrm{~g}$, independientemente de la edad gestacional(6), se ha identificado como uno de los principales factores de riesgo para la mortalidad infantil, especialmente de la neonatal(3-5). En el contexto individual, el BPN aumenta el riesgo de infecciones, desnutrición, parálisis infantil, deficiencias mentales, trastornos del aprendizaje, patologías cardiovasculares, enfermedades metabólicas, entre otras ${ }^{(7)}$. Es un problema de salud pública presente en los cinco continentes $^{(8)}$ que, finalmente, se relaciona con los principales mecanismos de transmisión intergeneracional de la pobreza y la desigualdad(9).

Las causas del BPN son multifactoriales, se trata de una interacción compleja entre factores fetales, maternos y socioeconómicos(10). Entre las causas maternas, se encuentra la desnutrición, la anemia, el control prenatal inadecuado, el uso y abuso de medicamentos y de sustancias ilícitas y tóxicas, las complicaciones obstétricas, la maternidad en edades extremas y el intervalo intergenésico corto(11).

Durante el año 2013, el Sistema de Vigilancia Nutricional de la Secretaría de Salud de Pasto, notificó que el $10,3 \%$ de todos los neonatos del municipio había presentado BPN, una tasa superior en un $28,8 \%$ a la reportada en 2010 para el país en general, cuando esa situación se presentó en el 8,0\% de los nacidos vivos $(\mathrm{NV})^{(12) .}$

De otro lado, la Seguridad Alimentaria y Nutricional (SAN) hace referencia a la disponibilidad suficiente y estable de alimentos, el acceso y el consumo oportuno y permanente de los mismos en cantidad, calidad e inocuidad por parte de todas las personas(13). Entre las principales causas de inseguridad alimentaria, se ha documentado la pobreza, ausencia de soberanía alimentaria, los elevados precios de los alimentos, la degradación del medio ambiente, la ineficiencia de la producción y distribución de alimentos, la injusticia social y la inequidad, entre otros(14).

Debido a los requerimientos nutricionales propios del periodo de crecimiento y desarrollo al inicio de la vida, la seguridad alimentaria en el hogar juega un papel determinante, no sólo del estado nutricional de los recién nacidos, sino en su potencial cognitivo, socio-emocional y motor(15). La seguridad alimentaria no depende exclusivamente de las condiciones propias de las familias, sino que en parte se explica por las características del contexto geográfico(16). Colombia ha experimentado un importante desarrollo social y económico, sin embargo el país se ha caracterizado por la magnitud en las desigualdades socioeconómicas en su interior(17,18); en el caso específico del departamento de Nariño, sistemáticamente se han reconocido las dificultades de índole económico y social, dada la marginación en que el gobierno central ha mantenido al territorio costero y limítrofe del país, con un pobre desarrollo industrial, alto desempleo e incompetencia productiva(19). Esta situación de pobreza conlleva a implicaciones perjudiciales para la región y sus habitantes, entre ellas, la inseguridad alimentaria de los hogares.

Si bien las características clínicas relacionadas con el BPN han sido reiteradamente analizadas(20), se hace necesario trascender y estudiar los determinantes socioeconómicos que inciden en la salud neonatal. El objetivo de este trabajo fue determinar la asociación entre la inseguridad alimentaria en el hogar durante el último trimestre del embarazo y el BPN en partos atendidos en el Hospital San Pedro de Pasto en un periodo de seis meses (septiembre - 2015 a febrero 2016).

\section{Materiales y métodos}

Estudio observacional, analítico, con diseño de casos y controles. La población de estudio estuvo constituida por recién nacidos a término en el Hospital San Pedro de Pasto, institución de referencia para la atención neonatal en la región, así, al tomar de allí tanto casos como controles, se garantizó la selección de la misma base poblacional.

Se consideró caso a todo recién nacido a término (con mínimo 37 semanas de gestación), con peso inferior a 2.500 g en su primer día de vida; el peso y la edad 
gestacional se obtuvieron de los registros de la historia clínica. Se excluyeron aquellos que no tenían autorización escrita por parte del adulto responsable, a los neonatos fruto de embarazos múltiples y a quienes presentaban patologías que pudieran alterar la medición del peso (alteraciones genéticas, malformaciones o tumoraciones uterinas). Los controles fueron recién nacidos a término, con peso mayor de 2.500 g y menor a 4.000 g en su primer día de vida, con los mismos criterios de exclusión determinados para los casos. Para cada caso se seleccionó como control el siguiente recién nacido en el Hospital.

Se utilizó una muestra por conveniencia, tomando todos los casos que se presentaron en el Hospital durante el periodo de observación e igual número de controles. Se recalculó la potencia considerando como parámetros un OR esperado de 5,0, una proporción de BPN en expuestos del $20 \%$, un control por cada caso, una confianza del $95 \%$ y una muestra total de 146 neonatos, para una potencia final del 80,5\%. Este procedimiento se llevó a cabo a través de la calculadora epidemiológica Epidat versión 4.1.

Se usaron fuentes primarias y secundarias de información, consistentes en encuestas, consulta de historias clínicas y del formato de BPN del Instituto Nacional de Salud -INS 110-. Se aplicó la Escala Latinoamericana y del Caribe para la medición de la Seguridad Alimentaria (ELCSA) validada en Colombia(21). Dicho instrumento consta de 15 variables, con opciones de respuesta sí o no; cinco ítems afirmativos corresponden a inseguridad alimentaria leve; entre seis y diez, inseguridad moderada; y diez ítems o más, inseguridad alimentaria severa; tal como fue validada y con el propósito de controlar el sesgo de memoria, se preguntó por la situación alimentaria del hogar durante los tres meses anteriores a la encuesta. Debido a que la recolección de información se realizó al momento del nacimiento, se garantiza que la exposición antecedió el desenlace.

Se describieron las variables sociales, demográficas y clínicas a través de tablas y estadísticos de resumen. Se comprobó normalidad con la prueba Shapiro Wilk. Para la comparación entre los grupos se utilizó la prueba $\chi^{2}$ de independencia de Pearson, prueba exacta de Fisher, $\mathrm{T}$ de Student para diferencia de medias con varianzas iguales y U de Mann Whitney.
Se construyó un modelo de regresión logística binaria para determinar las variables que maximizaban la probabilidad de ocurrencia del BPN, utilizando el método de pasos sucesivos y considerando como variables candidatas aquellas que contaban con plausibilidad biológica según la literatura y que cumplieron con el criterio de Hosmer y Lemeshow ( $\mathrm{p} \leq 0,25$ en el análisis bivariado).

\section{Consideraciones éticas}

Los principios éticos estuvieron siempre en concordancia con la declaración de Helsinki y la Resolución 8430 de 1993. Esta investigación fue clasificada como sin riesgo y avalada por el Comité de Ética en Investigación de la Universidad CES. Todos los representantes legales de los neonatos firmaron el consentimiento informado, siempre se respetó la autonomía y el secreto médico. La información fue almacenada y digitalizada en MS Office Excel 2007, luego exportada y procesada en SPSS versión 19, licencia de la Universidad CES de Medellín. Además, se solicitó el visto bueno de las directivas administrativas del Hospital San Pedro para la recolección de los datos y la realización del estudio.

\section{Resultados}

Durante los seis meses de observación, en el Hospital fueron identificados 73 niños a término con BPN (casos), a quienes se les buscó un número igual de controles. Los recién nacidos fueron principalmente varones $(54,1 \%)$, la mayoría de las familias residían en viviendas de estratos socioeconómicos bajos, la edad promedio de las madres fue 25,3 años, mientras que el $50 \%$ de las madres tenían como máximo nivel de formación académica, la secundaria, sin diferencias estadísticas entre casos y controles. Más de la mitad de las madres tenía un solo hijo, siendo mayor la proporción para el grupo de BPN sin diferencias estadísticas (Tabla 1).

\section{Características clínicas de las madres y de los recién nacidos}

Se observaron diferencias significativas entre los grupos en cuanto a condiciones clínicas de la madre durante el embarazo, como hipertensión arterial (HTA), vaginosis, ruptura prematura de membranas (RPM) y malnutrición por exceso. En el grupo de casos se presentó mayor proporción de preclamsia y de infecciones vaginales (Tabla 2). 
Tabla 1. Distribución de las características sociales, demográficas y clínicas de los recién nacidos en el Hospital San Pedro y sus madres, según grupo de estudio. Pasto, 2015 - 2016

\begin{tabular}{|c|c|c|c|c|}
\hline & Casos & Controles & Total & \\
\hline \multicolumn{5}{|l|}{ Sexo del niño n (\%) } \\
\hline Hombre & $39(53,4)$ & $40(54,8)$ & $79(54,1)$ & \multirow{2}{*}{$0,868^{1}$} \\
\hline Mujer & $34(46,6)$ & $33(45,2)$ & $67(45,9)$ & \\
\hline \multicolumn{5}{|c|}{ Estrato socioeconómico de la vivienda n (\%) } \\
\hline Estrato 1 & $56(76,7)$ & $55(75,3)$ & $111(76)$ & \multirow{3}{*}{$0,515^{1}$} \\
\hline Estrato 2 & $9(12,3)$ & $10(13,7)$ & $19(13)$ & \\
\hline Estrato $3,4 .^{3}$ & $8(11)$ & $8(11)$ & $16(11)$ & \\
\hline \multicolumn{5}{|c|}{ Escolaridad de la madre n (\%) } \\
\hline Ninguna o primaria & $19(27,4)$ & $21(28,8)$ & $40(28,1)$ & \multirow{3}{*}{$0,678^{1}$} \\
\hline Secundaria & $36(49,3)$ & $37(50,7)$ & $73(50)$ & \\
\hline Técnico o superior & $18(24,7)$ & $15(20,5)$ & $33(22,6)$ & \\
\hline \multicolumn{5}{|c|}{ Número de hijos de la madre $n(\%)$} \\
\hline Uno & $47(64,5)$ & $37(50,7)$ & $84(57,5)$ & \multirow{3}{*}{$0,115^{2}$} \\
\hline Dos & $19(26)$ & $27(37)$ & $46(31,5)$ & \\
\hline Tres o más ${ }^{3}$ & $7(9,5)$ & $9(12,3)$ & $16(11)$ & \\
\hline \multicolumn{5}{|c|}{ Antecedente de violencia n (\%) } \\
\hline $\mathrm{Si}$ & $9(12,3)$ & $5(6,8)$ & $14(9,6)$ & \multirow{2}{*}{$0,261^{1}$} \\
\hline No & $64(87,7)$ & $68(93,2)$ & $132(90,4)$ & \\
\hline \multicolumn{5}{|c|}{ Edad de la madre (años) } \\
\hline Media (DE) & $24,8(7,1)$ & $25,7(7,2)$ & $25,3(7,1)$ & \multirow{4}{*}{0,387} \\
\hline $\mathrm{Me}(\mathrm{Rq})$ & $23,0(12,0)$ & $25,0(11,0)$ & $24,0(11,0)$ & \\
\hline Mín- Máx & $15-42$ & $13-43$ & $13-43$ & \\
\hline $\mathrm{CV} \%$ & $28,4 \%$ & $27,9 \%$ & $28,2 \%$ & \\
\hline \multicolumn{5}{|c|}{ Talla de la madre $(\mathrm{m})$} \\
\hline Media (DE) & $1,54(0,06)$ & $1,55(0,06)$ & $1,55(0,06)$ & \multirow{4}{*}{$0,197^{4}$} \\
\hline $\mathrm{Me}(\mathrm{Rq})$ & $1,54(0,08)$ & $1,55(0,08)$ & $154(0,07)$ & \\
\hline Mín - Máx & $1,41-1,67$ & $1,42-1,68$ & $1,41-1,68$ & \\
\hline $\mathrm{CV} \%$ & $3,6 \%$ & $3,5 \%$ & $3,6 \%$ & \\
\hline \multicolumn{5}{|c|}{ Índice de Masa Corporal de la madre } \\
\hline Media (DE) & $22,83(2,94)$ & $24,08(3,58)$ & $23,45(3,32)$ & \multirow{4}{*}{0,022} \\
\hline $\mathrm{Me}(\mathrm{Rq})$ & $22,64(3,22)$ & $24,12(5,22)$ & $23,14(4,51)$ & \\
\hline Mín - Máx & $17,3-32,9$ & $17,4-33,6$ & $17,3-33,6$ & \\
\hline CV\% & $12,8 \%$ & $14,9 \%$ & $14,2 \%$ & \\
\hline \multicolumn{5}{|c|}{ Talla del recién nacido $(\mathrm{cm})$} \\
\hline Media (DE) & $46,33(2,42)$ & $49,14(1,99)$ & $47,73(2,62)$ & \multirow{4}{*}{$0,0001^{2}$} \\
\hline $\operatorname{Me}(\mathrm{Rq})$ & $47(3)$ & $49(3)$ & $48(4)$ & \\
\hline Mín- Máx & $38-52$ & $44-53$ & $38-53$ & \\
\hline $\mathrm{CV} \%$ & $5,2 \%$ & $4 \%$ & $5,5 \%$ & \\
\hline \multicolumn{5}{|c|}{ Perímetro cefálico del recién nacido $(\mathrm{cm})$} \\
\hline Media (DE) & $32,18(1,59)$ & $34,22(1,25)$ & $33,2(1,76)$ & \multirow{4}{*}{$<0,0001^{2}$} \\
\hline $\mathrm{Me}(\mathrm{Rq})$ & $32,5(1,5)$ & $34(1,5)$ & $33,45(2)$ & \\
\hline Mín - Máx & $26,5-34,5$ & $31,5-37,0$ & $26,5-37,0$ & \\
\hline $\mathrm{CV} \%$ & $4,9 \%$ & $3,7 \%$ & $5,3 \%$ & \\
\hline \multicolumn{5}{|c|}{ Peso del recién nacido $(\mathrm{g})$} \\
\hline Media (DE) & $2.201(276,8)$ & $3.055(310,8)$ & $2, .628$ (519) & \multirow{4}{*}{$<0,0001^{2}$} \\
\hline $\mathrm{Me}(\mathrm{Rq})$ & $2.300(300)$ & $3.030(465)$ & $2502(744)$ & \\
\hline Mín - Máx & $1.150-2.495$ & $2.510-3.860$ & $1150-3860$ & \\
\hline $\mathrm{CV} \%$ & $12,6 \%$ & $10,2 \%$ & $19,7 \%$ & \\
\hline
\end{tabular}


Tabla 2. Distribución de algunos antecedentes clínicos y obstétricos de las madres, según grupo de estudio

\begin{tabular}{|c|c|c|c|c|}
\hline & $\begin{array}{l}\text { Casos } \\
\text { n (\%) }\end{array}$ & $\begin{array}{c}\text { Controles } \\
\text { n (\%) }\end{array}$ & $\begin{array}{l}\text { Total } \\
\text { n (\%) }\end{array}$ & $\mathbf{p}$ \\
\hline \multicolumn{5}{|c|}{ Anemia } \\
\hline $\mathrm{Si}$ & $13(17,8)$ & $10(13,7)$ & $23(15,8)$ & \multirow{2}{*}{$0,496^{1}$} \\
\hline No & $60(82,2)$ & $63(86,3)$ & $123(84,2)$ & \\
\hline \multicolumn{5}{|c|}{ Hipertensión arterial } \\
\hline $\mathrm{Si}$ & $19(26)$ & $5(6,8)$ & $24(16,4)$ & \multirow{2}{*}{$0,002^{1}$} \\
\hline No & $54(74)$ & $68(93,2)$ & $122(83,6)$ & \\
\hline \multicolumn{5}{|c|}{ Amenaza de parto prematuro } \\
\hline $\mathrm{Si}$ & $31(42,5)$ & $20(27,4)$ & $51(34,9)$ & \multirow{2}{*}{$0,056^{1}$} \\
\hline No & $42(57,5)$ & $53(72,6)$ & $95(65,1)$ & \\
\hline \multicolumn{5}{|c|}{ Infección urinaria } \\
\hline $\mathrm{Si}$ & $46(63)$ & $40(54,8)$ & $86(58,9)$ & \multirow{2}{*}{$0,313^{1}$} \\
\hline No & $27(37)$ & $33(45,2)$ & $60(41,1)$ & \\
\hline \multicolumn{5}{|c|}{ Infección vaginal } \\
\hline $\mathrm{Si}$ & $40(54,8)$ & $21(28,8)$ & $61(41,8)$ & \multirow{2}{*}{$0,001^{1}$} \\
\hline No & $33(45,2)$ & $52(71,2)$ & $85(58,2)$ & \\
\hline \multicolumn{5}{|c|}{ Ruptura prematura de membranas } \\
\hline $\mathrm{Si}$ & $3(4,1)$ & $10(13,7)$ & $13(8,9)$ & \multirow{2}{*}{$0,042^{1}$} \\
\hline No & $70(95,9)$ & $63(86,3)$ & $133(91,1)$ & \\
\hline \multicolumn{5}{|c|}{ Actividad física fuerte } \\
\hline $\mathrm{Si}$ & $15(20,5)$ & $14(19,2)$ & $29(19,9)$ & \multirow{2}{*}{$0,836^{1}$} \\
\hline No & $58(79,5)$ & $59(80,8)$ & $117(80,1)$ & \\
\hline \multicolumn{5}{|c|}{ Sobrepeso u obesidad } \\
\hline $\mathrm{Si}$ & $15(20,5)$ & $29(39,7)$ & $44(30,1)$ & \multirow{2}{*}{$0,012^{1}$} \\
\hline No & $58(79,5)$ & $44(60,3)$ & $102(69,9)$ & \\
\hline \multicolumn{5}{|c|}{ Desnutrición materna } \\
\hline $\mathrm{Si}$ & $7(9,6)$ & $6(8,2)$ & $13(8,9)$ & \multirow{2}{*}{$0,771^{1}$} \\
\hline No & $66(90,4)$ & $67(91,8)$ & $133(91,1)$ & \\
\hline \multicolumn{5}{|c|}{ Consumo de alcohol, tabaco o drogas } \\
\hline $\mathrm{Si}$ & $2(2,7)$ & $2(2,7)$ & $4(2,7)$ & \multirow{2}{*}{$1,000^{1}$} \\
\hline No & $71(97,3)$ & $71(97,3)$ & $142(97,3)$ & \\
\hline \multicolumn{5}{|c|}{ Antecedente de aborto } \\
\hline $\mathrm{Si}$ & $10(13,7)$ & $8(11)$ & $18(12,3)$ & \multirow{2}{*}{$0,615^{1}$} \\
\hline No & $63(86,3)$ & $65(89)$ & $128(87,7)$ & \\
\hline \multicolumn{5}{|c|}{ Intervalo intergenésico menor a 2 años } \\
\hline $\mathrm{Si}$ & $1(1,4)$ & $2(2,7)$ & $3(2,1)$ & $0,560^{1}$ \\
\hline No & $2(98,6)$ & $71(97,3)$ & $143(97,9)$ & $1,000^{2}$ \\
\hline \multicolumn{5}{|c|}{ Recibió micronutrientes durante el embarazo } \\
\hline $\mathrm{Si}$ & $68(93,2)$ & $72(98,6)$ & $140(95,9)$ & $0,095^{1}$ \\
\hline No & $5(6,8)$ & $1(1,4)$ & $6(4,1)$ & $0,209^{2}$ \\
\hline Con & & & & \\
\hline $\mathrm{Si}$ & $6(8,2)$ & $10(13,7)$ & $16(11)$ & $0289^{1}$ \\
\hline No & $67(91,8)$ & $63(86,3)$ & $130(89)$ & $0,289^{1}$ \\
\hline Men & & & & \\
\hline $\mathrm{Si}$ & $16(21,9)$ & $8(11)$ & $24(16,4)$ & $0740^{1}$ \\
\hline No & $57(78,1)$ & $65(89)$ & $122(83,6)$ & $0, / 40$ \\
\hline
\end{tabular}

${ }^{1}$ Prueba $\mathrm{X}^{2}$ de independencia de Pearson

${ }^{2}$ Prueba exacta de Fisher 


\section{Seguridad alimentaria en el hogar}

El 58,9\% de los hogares presentó inseguridad alimentaria moderada o severa (Figura 1). Este fenómeno se observó con mayor frecuencia entre los casos $(83,6 \%$ vs $34,2 \%)$. Así, por cada hogar con inseguridad alimentaria en los controles, se presentaron aproximadamente 10 hogares con inseguridad alimentaria entre los casos (OR=9,8, IC95\% 4,2 23,3). Como la cantidad de casos y controles clasificados con inseguridad moderada y severa fue baja, esta variable fue dicotomizada como presencia o ausencia de inseguridad alimentaria en el hogar.

Factores sociales, demográficos, clínicos y de la seguridad alimentaria relacionados con el BPN

Se encontró asociación estadística entre el BPN y la inseguridad alimentaria, hipertensión arterial (HTA), vaginosis y malnutrición por exceso (sobrepeso $\mathrm{u}$ obesidad materna). La medida de asociación más alta (OR crudo) se observó en la inseguridad alimentaria (Tabla 3), es decir que la probabilidad de presentar BPN es 9,8 veces mayor entre los hogares con inseguridad alimentaria. De otro lado, la posibilidad de BPN cuando la madre presentó preclampsia, fue
4,0 veces mayor con respecto a la madre que no sufrió esta alteración $(\mathrm{OR}=5,0)$, un mayor odds se presentó entre los neonatos cuyas madres no tenían exceso de peso (sugiriendo un posible efecto protector).

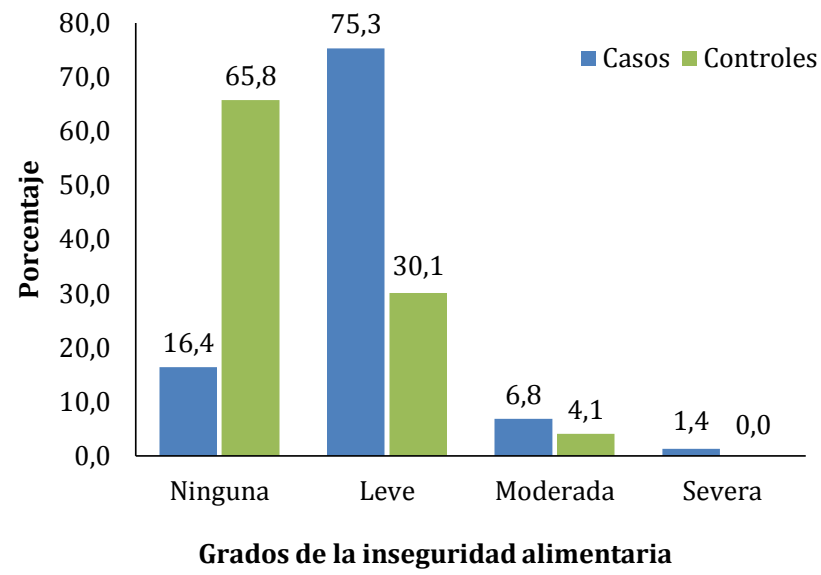

Figura 1. Distribución porcentual de la inseguridad alimentaria según grupo de estudio.

La proporción de inseguridad alimentaria (tanto leve como moderada y severa) es mayor entre los casos en comparación a los controles. Prueba $\chi^{2}$ de independencia de Pearson: $37,2(p=<0,0001)$

Tabla 3. Características sociales, demográficas y clínicas asociadas al bajo peso al nacer

\begin{tabular}{|c|c|c|c|c|c|c|c|}
\hline \multirow{2}{*}{ Variable } & \multicolumn{2}{|c|}{ Casos } & \multicolumn{2}{|c|}{ Controles } & \multirow{2}{*}{ OR } & \multirow{2}{*}{$p^{1}$} & \multirow{2}{*}{ IC $95 \%$ OR } \\
\hline & $n=73$ & $\%$ & $n=73$ & $\%$ & & & \\
\hline Inseguridad alimentaria & 61 & 83,6 & 25 & 34,2 & 9,8 & $<0,0001$ & $4,45-21,41$ \\
\hline Control prenatal tardío & 6 & 8,2 & 10 & 13,7 & 0,6 & 0,294 & $0,19-1,64$ \\
\hline Menos de 5 Controles Prenatales ${ }^{2}$ & 16 & 21,9 & 8 & 10,9 & 2,3 & 0,079 & $0,90-5,72$ \\
\hline Recibió micronutrientes & 5 & 6,8 & 1 & 1,37 & 5,3 & 0,133 & $0,60-46,48$ \\
\hline Procedencia urbana & 24 & 32,9 & 30 & 41,1 & 0,7 & 0,304 & $0,36-1,38$ \\
\hline Anemia & 60 & 82,2 & 63 & 86,3 & 1,4 & 0,497 & $0,56-3,35$ \\
\hline Intervalo intergenésico $<2$ años & 1 & 1,37 & 2 & 2,74 & 0,5 & 0,567 & $0,04-5,56$ \\
\hline Amenaza parto pretérmino & 31 & 42,5 & 20 & 27,4 & 2,0 & 0,058 & $0,98-3,91$ \\
\hline Hipertensión arterial & 19 & 26 & 5 & 6,8 & 4,8 & 0,003 & $1,68-13,65$ \\
\hline Infección urinaria materna & 46 & 63 & 40 & 54,8 & 1,4 & 0,313 & $0,73-2,73$ \\
\hline Vaginosis & 40 & 54,8 & 21 & 28,8 & 3,0 & 0,002 & $1,51-5,95$ \\
\hline Violencia & 9 & 12,3 & 5 & 6,8 & 1,9 & 0,267 & $0,61-6,01$ \\
\hline Antecedente de RPM ${ }^{3}$ & 10 & 13,7 & 3 & 4,1 & 0,3 & 0,054 & $0,07-1,02$ \\
\hline Desnutrición materna & 5 & 6,8 & 3 & 4,1 & 1,2 & 0,772 & $0,38-3,71$ \\
\hline Exceso de peso en la madre & 15 & 20,5 & 29 & 39,7 & 0,4 & 0,013 & $0,19-0,82$ \\
\hline Nivel bajo de educación & 19 & 26 & 21 & 28,8 & 0,9 & 0,711 & $0,42-1,80$ \\
\hline Estrato socioeconómico bajo & 56 & 76,7 & 55 & 75,3 & 1,1 & 0,846 & $0,50-2,31$ \\
\hline Actividad física fuerte & 15 & 20,5 & 14 & 19,2 & 1,1 & 0,836 & $0,48-2,46$ \\
\hline Antecedente de aborto & 10 & 13,7 & 8 & 10,9 & 1,3 & 0,615 & $0,48-3,48$ \\
\hline
\end{tabular}

${ }^{1}$ Prueba $\mathrm{X}^{2}$ de independencia de Pearson. ${ }^{2}$ Control prenatal. ${ }^{3}$ Ruptura prematura de membranas. OR crudos 


\section{Características que mejor explican la presencia de BPN}

Se construyó un modelo de regresión logística binario incondicional y explicativo, considerando como exposición la inseguridad alimentaria y como variables de ajuste tener menos de cinco controles prenatales, recibir micronutrientes durante el embarazo, precedente de amenaza de parto pretérmino, HTA, vaginosis, antecedentes de RPM y exceso de peso en la madre. Entre los modelos construidos se eligió el más parsimonioso, este modelo incluyó la inseguridad alimentaria, HTA, vaginosis, antecedente de RPM y exceso de peso en la madre, y explicó el 54,7\% de la variación en el logit de presentar BPN.

Después del ajuste, la medida de asociación entre BPN e inseguridad alimentaria aumentó drásticamente (OR crudo: 9,8, OR ajustado: 19,9), lo cual indicaría una subestimación del efecto de la inseguridad alimentaria en el análisis crudo (Tabla 4).

Tabla 4. Modelo de regresión logística binaria incondicional para el BPN en función de la inseguridad alimentaria, ajustado por factores clínicos de la madre

\begin{tabular}{|c|c|c|c|c|c|c|c|c|}
\hline & \multirow{2}{*}{$\begin{array}{c}\text { OR } \\
\text { crudo }\end{array}$} & \multicolumn{2}{|c|}{ IC $95 \%$} & \multirow{2}{*}{$\begin{array}{c}\text { OR } \\
\text { ajustado }\end{array}$} & \multirow{2}{*}{$\begin{array}{c}\text { Error } \\
\text { estándar }\end{array}$} & \multirow{2}{*}{$\mathbf{p}$} & \multicolumn{2}{|c|}{ IC $95 \%$} \\
\hline & & Inferior & Superior & & & & Inferior & Superior \\
\hline Inseguridad alimentaria & 9,8 & 4,5 & 21,4 & 19,9 & 0,53 & $<0,0001$ & 7,0 & 56,7 \\
\hline Hipertensión arterial & 4,8 & 1,7 & 13,7 & 10,5 & 0,71 & 0,001 & 2,6 & 41,8 \\
\hline Antecedentes de RPM & 0,3 & 0,1 & 1,0 & 0,05 & 0,84 & 0,001 & 0,0 & 0,29 \\
\hline Sobrepeso u obesidad & 0,4 & 0,2 & 0,8 & 0,24 & 0,52 & 0,007 & 0,1 & 0,67 \\
\hline Vaginosis & 3,0 & 1,5 & 6,0 & 4,00 & 0,48 & 0,005 & 1,5 & 10,44 \\
\hline Constante & & & & 0,12 & & & & \\
\hline
\end{tabular}

$\mathrm{R}^{2}$ de Nagelkerke 0,547

\section{Discusión}

La inseguridad alimentaria en el hogar durante el último trimestre del embarazo se asoció fuertemente con el BPN en el área de influencia del Hospital San Pedro de Pasto durante el periodo de estudio. La medida del efecto crudo y ajustado difirió; el OR aumentó en un 97,8\% después de considerar características clínicas del embarazo, así, la posibilidad de presentar BPN cuando existe inseguridad alimentaria en el hogar es 19,9 veces en comparación con dicha posibilidad en un hogar sin inseguridad.

Algunos estudios asocian el BPN con factores socioeconómicos y nutricionales como el ingreso económico en el hogar, la calidad, cantidad de alimentos para la familia, y el nivel de educación de los padres. Demelash, et al.(22), encontraron que el BPN era explicado por un ingreso mensual inferior a \$26 US (OR: 3,8; IC del 95\%: 1,54-9,41) y la falta de educación formal de los padres (OR: 6; IC del 95\%: 1,34 a 26,90). Por su parte, Misra et al., afirman que la calidad y cantidad de la dieta materna durante el embarazo son determinantes importantes de peso de sus hijos al nacer(23); mientras que Kader y Perera concluyeron que la educación materna, el estado nutricional y la atención prenatal son determinantes clave en la ocurrencia del BPN(24).

La influencia de la nutrición materna sobre el peso al nacer ha sido estudiada en diferentes contextos y poblaciones. Los resultados han sido consistentes en mostrar la necesidad de un nivel mínimo de nutrientes requeridos para obtener un peso adecuado al nacer y una mayor impacto de las intervenciones nutricionales durante el embarazo, en contraste con las ejecutadas en otros momentos del ciclo vital de la madre(25-27).

En el presente estudio, la HTA de la madre se asoció al BPN, lo que concuerda con la literatura revisada; Bello, et al.(28), encontraron que la preeclampsia (OR: 3,2 ; IC 95\%: 2,1; 5,1) estaba asociada al BPN, igual que Bramham et al.(29), quienes en un metaanálisis encontraron asociación entre la hipertensión crónica y el BPN (RR: 3,2; IC 95\% 2,2; 4,4). La principal explicación fisiopatológica establece que el bajo flujo sanguíneo crónico en el lecho placentario materno y en el feto durante la vida intrauterina, implica a largo plazo un pobre desarrollo fetal y BPN(28). 
En el caso de la infección vaginal, se ha encontrado en la literatura una conexión con el BPN, igual que en esta investigación. Thorsen, et al.,(30), lo reportaron en primigestantes danesas (OR: 4,3 IC 95\%: 1,5 - 12); al igual que Afolabi, et al.,(31), quienes en un estudio prospectivo hallaron que la vaginosis se asocia significativamente con el BPN, tanto en recién nacidos pretérmino como a término (RR, 3,20; IC del 95\%, 1,29-7,94), una posible explicación sería la presencia de citoquinas proinflamatorias asociadas a la infección, que podrían inducir fiebre y generar pérdida de peso.

Por su parte, en la literatura se ha asociado la RPM con el BPN. Muchemi, et al.(32), (OR: 2,95; IC 95\%: 1,14 - 7,62) propusieron como posible mecanismo causal la presencia de infecciones vaginales como variable intermedia entre la RPM y el BPN. Afolabi, et al.(31), encontraron que por cada caso de BPN en mujeres sin alteraciones, aproximadamente siete recién nacidos con presentaron RPM (RR: 6,75; IC del 95\%, 3,1114,67). Contrario a los anteriores hallazgos, en este estudio se evidenció una posible asociación protectora para la cual no se ha encontrado una explicación plausible, sin embargo, entre las hipótesis están la inclusión exclusiva de neonatos a término, que la mayoría de las pacientes incluidas y que cursaron con RPM, se hospitalizaron para retener el feto hasta llegar a mejor peso y edad gestacional; además porque esta RPM sucedió de manera tardía en la gestación.

De otro lado, se ha reportado que el exceso de peso materno podría generar recién nacidos grandes para la edad gestacional y macrosomía (OR: 1,45, IC 95\% $1,29$ - 1,63 y OR: 1,88, IC: $1,67-2,11)^{(33)}$. En este estudio también se encontró una posible asociación protectora, esta situación podría explicarse porque el feto recibiría los nutrientes suficientes requeridos de parte de su madre con sobrepeso u obesidad y por la carga alta y continua de glucosa que usualmente circula en la sangre de las personas con exceso de peso.

No se encontró asociación entre el BPN y el área de residencia, contrario a lo detallado en la literatura, donde evidenciaron un mayor riesgo de BPN entre quienes residen en el área urbana (OR: 2,1; 95 \% CI: 1,04-4,33)(22). Una posible explicación es que, a pesar de que la institución atiende todos los regímenes del sistema de salud, la población pertenece mayoritariamente a estratos socioeconómicos bajos de Pasto, con relativamente baja afluencia de personas del área rural, y esta condición se presentó en los casos como en los controles.

Tampoco se encontró asociación entre anemia materna y BPN, similar a la investigación realizada por Koura, et al.(34), en África, pero contrario a lo reportado por Misra, et al.(23), en una revisión sistemática con metaanálisis, se encontró asociación entre la anemia materna en el primer trimestre de gestación y el BPN; sin embargo, esta asociación no fue consistente para el segundo y tercer trimestre de gestación $^{35}$. En este estudio, más del $80 \%$ de las maternas tuvieron anemia durante la gestación (casos $82,2 \%$ y controles $86,3 \%$ ), una proporción alta en ambos grupos, lo cual sugiere la necesidad de intervenciones de carácter individual y públicas para mejorar las condiciones nutricionales de las mujeres gestantes en el municipio de Pasto.

Existen pocos estudios donde se analiza la relación entre la inseguridad alimentaria y el BPN, algunos de ellos no son comparables debido a aspectos metodológicos. En Medellín, Colombia durante el año 2014, concluyeron que los ingresos económicos inferiores a un salario mínimo, se asociaba con neonatos pequeños para la edad gestacional y con $\mathrm{BPN}(36)$; argumentaron que la inseguridad alimentaria en el hogar limita el consumo de alimentos adecuados durante la gestación, incrementando el riesgo de deficiencia de micronutrientes y, por lo tanto, de BPN. De otro lado, en Pereira, Colombia, valoraron el estado nutricional y la percepción de inseguridad alimentaria en gestantes adolescentes, quienes indicaron inseguridad alimentaria en el 63,3\% de los casos y también encontraron asociación entre la inseguridad alimentaria y BPN(37); no obstante, los grupos de edad materna no son comparables con las personas estudiadas en este trabajo. Entre las limitaciones de este estudio, se encuentra la imposibilidad de medir la inseguridad alimentaria a través de indicadores objetivos; sin embargo, se resalta la utilización de un instrumento válido para Colombia y aplicable a la región latinoamericana.

Entre las fortalezas, se resalta la utilización de fuentes primarias de datos tanto para los casos como para los controles, recabadas en función de este proyecto. Adicionalmente, la recolección de información sobre seguridad alimentaria se realizó por parte del investigador, con formación en medicina y pediatría, 
acompañado de una profesional en psicología para mejorar la empatía y obtener datos confiables.

\section{Conclusión}

La inseguridad alimentaria en el hogar durante el último trimestre del embarazo se asoció con el BPN en el área de influencia del Hospital San Pedro de Pasto, siendo el principal factor determinante del BPN. Así, para intervenir de manera efectiva la morbimortalidad que conlleva el BPN, se recomienda priorizar las políticas tendientes a garantizar la seguridad alimentaria de la población vulnerable, principalmente en las mujeres en edad reproductiva y de las mujeres embarazadas.

\section{Conflicto de intereses}

No existen conflictos de intereses.

\section{Referencias}

1. Abdullah A, Hort K, Butu Y, Simpson L. Risk factors associated with neonatal deaths: a matched case-control study in Indonesia. Glob Health Action. 2016;9:30445. doi: 10.3402/gha.v9.30445

2. Deng Y, Xiong F, Wu MM, Yang F. A follow-up on first-year growth and development of 61 very low birth weight preterm infants. Zhongguo Dang Dai Er Ke Za Zhi Chin J Contemp Pediatr. 2016;18(6):482-7.

3. Hailu LD, Kebede DL. Determinants of Low Birth Weight among Deliveries at a Referral Hospital in Northern Ethiopia. BioMed Res Int [Internet]. 2018 [citado 11 de octubre de 2018]; 2018:1-8. Disponible en: https://www.ncbi.nlm.nih.gov/pmc/articles/PMC5937567

4. Levitzky O, Lerner-Geva L, Dollberg S, Reichman B. The Israel national very low birth weight infant database. Harefuah. 2016;155(1):32-6, 67.

5. Noureddine E, Abdellatif B. Prevalence and Determinants of Low Birth Weight: A Case-Control Study in Marrakesh (Morocco). Iran J Public Health. 2015;44(3):422-4.

6. Organización Mundial de la Salud. Metas mundiales de nutrición 2015. Documento normativo sobre bajo peso al nacer [Internet]. Ginebra: OMS; 2017 [citado 11 de octubre de 2018]. Disponible en: https://www.who.int/nutrition/publications/globaltargets 2025_policybrief_lbw/es/

7. Quintana V, Inés N, Zárraga MY, Luis J, Ávila Reyes R. Recién nacidos con bajo peso; causas, problemas y perspectivas a futuro. Bol Méd Hosp Infant México. 2004;61(1):73-86.

8. Mahumud RA, Sultana M, Sarker AR. Distribution and Determinants of Low Birth Weight in Developing Countries. J Prev Med Pub Health. 2017;50(1):18-28.

9. Unicef, CEPAL. Desnutrición infantil en América Latina y el Caribe. Desafíos. Boletín de la infancia y adolescencia sobre el avance de los ODM [Internet]. Santiago: Naciones Unidas; 2006 [citado 13 de noviembre de 2016]. Disponible en:
http://www.unicef.cl/archivos_documento/164/Desafios\% 202.pdf

10. Altenhöner $T$, Köhler M, Philippi M. The Relevance of Maternal Socioeconomic Characteristics for Low Birth Weight - a Case-Control Study. Geburtshilfe Frauenheilkd. 2016;76(3):248-54.

11. López JI, Lugones-Botell M, Pineda V, M L, Virella Blanco J. Algunos factores maternos relacionados con el bajo peso al nacer. Rev Cuba Obstet Ginecol. 2004;30(1):0-0.

12. Instituto Colombiano de Bienestar Familiar (ICBF). Encuesta Nacional de la Situación Nutricional en Colombia, 2010. Bogotá, Colombia: ICBF; 2011.

13. Consejo Nacional de Política Económica Social - República de Colombia, Departamento Nacional de Planeación. Documento Conpes Social 113. Política nacional de seguridad alimentaria y nutricional [Internet]. Bogotá: SIPI; 2008 [citado 13 de noviembre de 2016]. Disponible en: http://www.sipi.siteal.org/sites/default/files/sipi_normativ a/colombia_politica_nacional_de_seguridad_alimentaria_y_n utricional_psan_2008.pdf

14. Salvador-Castell G, de la Cruz JN, Pérez-Rodrigo C, Aranceta J. Escalas de evaluación de la inseguridad alimentaria en el hogar. Rev Esp Nutr Comunitaria. 2015;(2):270-276.

15. Ke J, Ford-Jones EL. Food insecurity and hunger: A review of the effects on children's health and behaviour. Paediatr Child Health. 2015;20(2):89-91.

16. Ma X, Liese AD, Bell BA, Martini L, Hibbert J, Draper C, et al. Perceived and geographic food access and food security status among households with children. Public Health Nutr. 2016;19(15):2781-8.

17. Colombia. Instituto Nacional de Salud, Observatorio Nacional de Salud. Informe Nacional de las Desigualdades Sociales en Salud en Colombia 2015 [Internet]. Bogotá: Imprenta Nacional de Colombia; 2015 [citado 2 de mayo de 2016]. Disponible

en: https://www.minsalud.gov.co/sites/rid/Lists/BibliotecaDig ital/RIDE/VS/ED/6to-informe-ons.pdf

18. Organización Mundial de la Salud. Estadísticas Sanitarias Mundiales 2014 [Internet]. Ginebra: OMS; 2014 [citado 11 de octubre de 2018]. Disponible en: http://www.who.int/gho/publications/world_health_statist ics/2014/es/

19. Instituto Departamental de Salud de Nariño. Plan de Salud Territorial «Salud para el buen vivir» 2012 - 2015. Nariño: Gobernación de Nariño; 2012.

20. Dayanithi M. Low birth weight and premature births and their associated maternal factors. Int J Community Med Public Health. 2018;5(6):2277.

21. Organización de las Naciones Unidas para la Alimentación y la Agricultura (FAO), Comité científico de la ELCSA. Escala Latinoamericana y Caribeña de Seguridad Alimentaria (ELCSA): Manual de uso y aplicaciones [Internet]. Roma: FA0; 2012 [citado 11 de octubre de 2018]. 78 p. Disponible en: http://www.fao.org/3/a-i3065s.pdf

22. Demelash H, Motbainor A, Nigatu D, Gashaw K, Melese A. Risk factors for low birth weight in Bale zone hospitals, South-East Ethiopia: a case-control study. BMC Pregnancy Childbirth. 2015;15:264.

23. Misra A, Ray S, Patrikar S. A longitudinal study to determine association of various maternal factors with neonatal birth weight at a tertiary care hospital. Med J Armed Forces India. 2015;71(3):270-3. 
24. Kader M, Perera N. Socio-economic and nutritional determinants of low birth weight in India. North Am J Med Sci. 2014;6(7):302-8.

25. Lechtig A, Yarbrough C, Delgado H, Habicht JP, Martorell R, Klein RE. Influence of maternal nutrition on birth weight. Am J Clin Nutr. 1975;28(11):1223-33.

26. Coelho Nde L, Cunha DB, Esteves AP, Lacerda EM, ThemeFilha MM. Dietary patterns in pregnancy and birth weight. Revista de Saúde Pública. 2015;49:62. doi: 10.1590/S00348910.2015049005403

27. Abubakari A, Jahn A. Maternal Dietary Patterns and Practices and Birth Weight in Northern Ghana. PLoS One. 2016;11(9):e01622885. 10.1371/journal.pone.0162285

28. Bello-Lujan LM, Saavedra-Santana P, Gutiérrez-García LE, García-Hernández JA, Serra-Majem L. Sociodemographic and health characteristics associated with low birth weight in de Canary Islands. Nutr Hosp. 2015;32(4):1541-7. doi: 10.3305/nh.2015.32.4.9456.

29. Bramham K, Parnell B, Nelson-Piercy C, Seed PT, Poston L, Chappell LC. Chronic hypertension and pregnancy outcomes: systematic review and meta-analysis. The BMJ. 2014;348(g2301). doi: 10.1136/bmj.g2301.

30. Thorsen P, Vogel I, Olsen J, Jeune B, Westergaard JG, Jacobsson $\mathrm{B}$, et al. Bacterial vaginosis in early pregnancy is associated with low birth weight and small for gestational age, but not with spontaneous preterm birth: a populationbased study on Danish women. J Matern-Fetal Neonatal Med. 2006;19(1):1-7.
31. Afolabi BB, Moses OE, Oduyebo 00. Bacterial Vaginosis and Pregnancy Outcome in Lagos, Nigeria. Open Forum Infect Dis. 2016;3(1):ofw030. doi: 10.1093/ofid/ofw030.

32. Muchemi OM, Echoka E, Makokha A. Factors associated with low birth weight among neonates born at Olkalou District Hospital, Central Region, Kenya. Pan Afr Med J. 2015;20:108. doi: 10.11604/pamj.2015.20.108.4831

33. Liu P, Xu L, Wang Y, Zhang Y, Du Y, Sun Y, et al. Association between perinatal outcomes and maternal pre-pregnancy body mass index. Obes Rev. 2016;17(11):1091-102. doi: 10.1111/obr.12455.

34. Koura GK, Ouedraogo S, Le Port A, Watier L, Cottrell G, Guerra J, et al. Anaemia during pregnancy: impact on birth outcome and infant haemoglobin level during the first 18 months of life. Trop Med Int Health TM IH. 2012;17(3):28391. doi: 10.1111/j.1365-3156.2011.02932.x.

35. Rahmati S, Delpishe A, Azami M, Hafezi-Ahmadi MR, Sayehmiri K. Maternal Anemia during pregnancy and infant low birth weight: A systematic review and Meta-analysis. Int J Reprod Biomed Yazd Iran. 2017;15(3):125-34.

36. Restrepo-Mesa SL, Zapata López N, Sosa P, Elena B, Vásquez E, Estela L, et al. Embarazo adolescente: características maternas y su asociación con el peso al nacer del neonato. Arch Latinoam Nutr. 2014;64(2):99-107.

37. Quintero Tabares R, Astudillo M, Nelcy M, Sierra Á, Elena L, Perea $M$, et al. Nutritional status and food safety in teenage pregnant women: Pereira, Colombia, 2009. Investig Educ En Enferm. 2010;28(2):204-13. 